\title{
Importance of considering methodological variables in rat studies
}

\section{Namrata Joshi}

\section{Dalhousie University}

Developmental stress exposure is a risk factor for developing a host of neuropsychiatric disorders in the future. ${ }^{1}$ Establishing causal links between developmental stress and such disorders necessitates the use of animal models. ${ }^{1}$ Although such studies attempt to use similar experimental designs to seek answers to similar questions, they often end up with divergent results. For example, a recent study examining effects of stress on hippocampal neurogenesis failed to replicate the results of a number of earlier studies; it was speculated that subtle differences in animal housing, handling or other methodological factors may have been responsible. ${ }^{2}$ Similarly, the effects of amygdalar lesions on activation of the body's main stress response system, the hypothalamic-pituitary-adrenal (HPA) axis, are reported to be divergent; again, the discrepancy was partly explained by factors such as choice of rat strain and animal housing conditions. ${ }^{3}$ My own research experiences with stress exposure in adolescent rats demonstrated similar divergence from published findings that can best be explained by subtle methodological differences between studies. This commentary discusses three issues related to animal breeding and rearing that may contribute to variability in outcomes when investigating stress in rats: the diurnal light cycle in the rats' housing environment, features of housing complexity such as cage size and population density, and features of animal transport prior to experimentation.

The first factor is the potential effect of the diurnal light cycle under which the experimental animals are housed. Rats, as nocturnal animals, are most active during their subjective night. Both basal ${ }^{4}$ and stress-induced activity of the HPA axis shows diurnal fluctuations. For example, repeated restraint stress induces a greater impact on food intake during the dark phase (night) of the light cycle of rats. ${ }^{5}$ Despite the potential for discrepant results, testing rats at different times in their circadian cycle occurs routinely in stress response research. For example, Bourke et al. ${ }^{6}$ and Yogarson et al. ${ }^{7}$ looked at the social rearing environment and anxiety-like behaviour during different phases of the diurnal cycle.

The second factor is the potential difference in home cage features of the experimental animals; two features are of primary importance. First, home cage dimensions and the number of rats housed in each cage dictate the volume of space available to each animal. These can differ between studies and since overcrowding is a significant stressor ${ }^{3}$, too little space can result in all animals, including those serving as controls, becoming stressed, thereby confounding differences between experimental and control groups. Secondly, the number of cage mates available may affect the social interacting, which can impact rat behaviour and physiology, especially in adolescent rats. ${ }^{6,7}$ Furthermore, the outcome of a stressor protocol can be affected by the degree of social support available in the form of cage mates. ${ }^{8}$ Thus, a seemingly simple decision such as how many animals to house together in a defined space can significantly affect the outcome of the study.

A third factor that may influence results of stress studies is animal transport and/or acclimatization before an experiment. Transport is a complex experience that can includes exposure to a complex array of physical, physiological, and psychological stressors. ${ }^{9}$ It is common practice to acclimatize transported animals for one week to ten days before experimental use and ideally, this would be long enough for stress response parameters and other experimental variables to return to a baseline level. Unfortunately, limited information is available on the ideal acclimatization time in rats. ${ }^{9}$ It is noteworthy that transportation conditions can even have a generational effect, as transporting pregnant animals can induce prenatal stress. The early environment is likewise important. ${ }^{10}$ Specific data on effects of transport 
C $\quad$ on various rat strains and sexes should be determined. Although sometimes more costly, breeding experimental animals in-house would allow researchers greater control over the pre-natal and early post-natal environments. It is, therefore, worth considering this option in studies that explore questions about the biology of the stress response.

Because the precise effects of each of the described factors is difficult to gauge, and rats can be exposed to multiple factors simultaneously, it is more practical to take these factors into account before an experiment begins. To this end, researchers should conduct studies on the effect of these issues on various stress-response parameters. Publication of reviews of the effects of such methodological factors on previously conducted research on stress would also prove to be beneficial. In a climate where funding for research is limited, it is vital that methodological questions be clearly addressed to facilitate replication of results.

\section{Acknowledgements}

The author would like to acknowledge the help of her supervisors, Drs. Tara Perrot and Ronald Leslie.

\section{References}

1. Teicher MH, Tomoda A, Andersen SL. Neurobiological consequences of early stress and childhood maltreatment: are results from human and animal studies comparable? Annals of the New York Academy of Sciences. 2006;1071:313-23. Epub 2006/08/08.

2. Hanson ND, Owens MJ, Boss-Williams KA, Weiss JM, Nemeroff CB. Several stressors fail to reduce adult hippocampal neurogenesis. Psychoneuroendocrinology. 2011;36(10):1520-9. Epub 2011/05/24.

3. Herman JP, Figueiredo $H$, Mueller NK, Ulrich-Lai $Y$, Ostrander MM, Choi DC, et al. Central mechanisms of stress integration: hierarchical circuitry controlling hypothalamo-pituitary-adrenocortical responsiveness. Frontiers in neuroendocrinology. 2003;24(3):151-80. Epub 2003/11/05.

4. Girotti M, Weinberg MS, Spencer RL. Differential responses of hypothalamuspituitary-adrenal axis immediate early genes to corticosterone and circadian drive. Endocrinology. 2007;148(5):2542-52. Epub 2007/02/17.

5. Rybkin, II, Zhou Y, Volaufova J, Smagin GN, Ryan DH, Harris RB. Effect of restraint stress on food intake and body weight is determined by time of day. The American journal of physiology. 1997;273(5 Pt 2):R1612-22. Epub 1997/12/31.

6. Bourke $\mathrm{CH}$, Neigh GN. Behavioral effects of chronic adolescent stress are sustained and sexually dimorphic. Hormones and behavior. 2011;60(1):11220. Epub 2011/04/07.

7. Yorgason JT, Espana RA, Konstantopoulos JK, Weiner JL, Jones SR. Enduring increases in anxiety-like behavior and rapid nucleus accumbens dopamine signaling in socially isolated rats. The European journal of neuroscience. 2013;37(6):1022-31. Epub 2013/01/09.

8. Ruis MA, te Brake JH, Buwalda B, De Boer SF, Meerlo P, Korte SM, et al. Housing familiar male wildtype rats together reduces the longterm adverse behavioural and physiological effects of social defeat. Psychoneuroendocrinology. 1999;24(3):285-300. Epub 1999/04/02.

9. Obernier JA, Baldwin RL. Establishing an appropriate period of acclimatization following transportation of laboratory animals. ILAR journal / National Research Council, Institute of Laboratory Animal Resources. 2006;47(4):364-9. Epub 2006/09/12.

10. Ward ID, Zucchi FC, Robbins JC, Falkenberg EA, Olson DM, Benzies K, et al. Transgenerational programming of maternal behaviour by prenatal stress. BMC pregnancy and childbirth. 2013;13 Suppl 1:S9. Epub 2013/03/06.

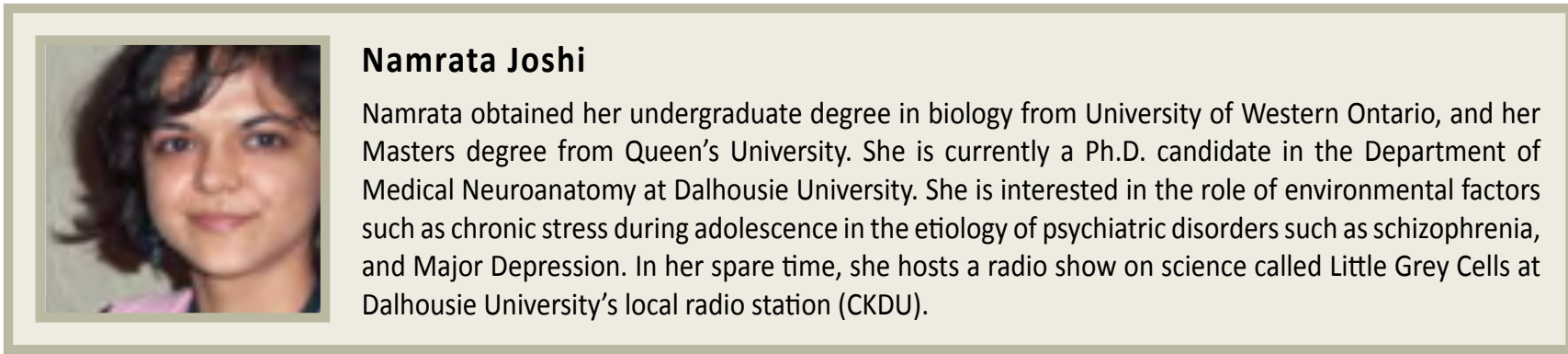

